\title{
Communications
}

Examens de spécialiste

Examen de spécialiste en vue de l'obtention du titre de spécialiste en chirurgie pédiatrique

Date:

les jeudi et vendredi $2 / 3$ décembre 2021

Lieu: Hôpital universitaire pédiatrique, Bâle

Délai d'inscription: 30 juin 2021

Vous trouverez de plus amples informations sur le site web de l'ISFM www.siwf.ch $\rightarrow$ formation postgraduée $\rightarrow$ Titres de spécialiste et formations approfondies $\rightarrow$ chirurgie pédiatrique

\section{Examen de spécialiste en vue de l'obtention du titre de spécialiste en angiologie}

Première partie (examen écrit)

Vendredi 3 décembre 2021, lors du 21 ${ }^{\mathrm{e}}$ Congrès de l'Union des Sociétés Suisses des Maladies Vasculaires, à Interlaken

Deuxième partie (examen oral et pratique)

Vendredi 17 décembre 2021, à l'HFR - Hôpital cantonal, Fribourg

\section{Délai d'inscription: 30 septembre 2021}

Vous trouverez de plus amples informations sur le site web de l'ISFM www.siwf.ch $\rightarrow$ formation postgraduée $\rightarrow$ Titres de spécialiste et formations approfondies $\rightarrow$ angiologie

\section{Examen pour l'obtention de l'attestation de formation complémentaire en phlébologie}

Examen écrit (première partie)

Date: le vendredi 3 décembre 2021

Lieu: Interlaken, lors du 21 ${ }^{\mathrm{e}}$ Congrès de l'Union des Sociétés Suisses des Maladies Vasculaires

Délai d'inscription: 17 septembre 2021

Inscription:

Dr Jürg Traber

Venenklinik Kreuzlingen

Brückenstrasse 9

CH-8280 Kreuzlingen

Tél. 0716782266

e-mail: j.traber[at]venenklinik.ch

Informations:

voir sous www.phlebology.ch,

link Certificat de compétence

Veuillez noter que l'examen est en anglais! 